\title{
Scaling behavior in crackle sound during lung inflation
}

\author{
Adriano M. Alencar, ${ }^{1,2}$ Zoltán Hantos, ${ }^{3}$ Ferenc Peták, ${ }^{3}$ József Tolnai, ${ }^{3}$ Tibor Asztalos, ${ }^{3}$ Stefano Zapperi, ${ }^{2,4}$ \\ José S. Andrade, Jr., ${ }^{4,5}$ Sergey V. Buldyrev, ${ }^{2}$ H. Eugene Stanley, ${ }^{2}$ and Béla Suki ${ }^{1}$ \\ ${ }^{1}$ Department of Biomedical Engineering, Boston University, Boston, Massachusetts 02215 \\ ${ }^{2}$ Center for Polymer Studies and Department of Physics, Boston University, Boston, Massachusetts 02215 \\ ${ }^{3}$ Department of Medical Informatics and Engineering, and Institute of Experimental Surgery, \\ Albert Szent-Gyorgyi Medical University, Szeged, Hungary \\ ${ }^{4}$ PMMH Ecole Superieure de Physique et Chimie Industrielles, 10 rue Vauquelin, 75231 Paris Cedex 05, France \\ ${ }^{5}$ Departamento de Física, Universidade Federal do Ceará, 60451-970 Fortaleza, Ceará, Brazil
}

(Received 6 April 1998; revised manuscript received 14 July 1999)

\begin{abstract}
During slow inflation of lung lobes, we measure a sequence of short explosive transient sound waves called "crackles," each consisting of an initial spike followed by ringing. The crackle time series is irregular and intermittent, with the number of spikes of size $s$ following a power law, $n(s) \propto s^{-\alpha}$, with $\alpha=2.77 \pm 0.05$. We develop a model of crackle wave generation and propagation in a tree structure that combines the avalanchelike opening of airway segments with the wave propagation of crackles in a tree structure. The agreement between experiments and simulations suggests that (i) the irregularities are a consequence of structural heterogeneity in the lung, (ii) the intermittent behavior is due to the avalanchelike opening, and (iii) the scaling is a result of successive attenuations acting on the sound spikes as they propagate through a cascade of bifurcations along the airway tree. [S1063-651X(99)13810-8]

PACS number(s): 87.19.- j, 43.25.+y
\end{abstract}

\section{INTRODUCTION}

There is much current interest in the puzzling physics associated with the lung [1-6]. In particular, the short "explosive" transient waves, called crackles, are not fully understood. Forgacs [7] proposed that crackles are associated with the sudden opening of closed airways. The discrete emission of crackles from the lung tissue has been modeled as a stress-relaxation quadrupole [8], and this work has received experimental support [9]. Also, gas trapping during inflation has been found to correlate with crackle sound intensity [10]. Characteristics of individual crackles have long been used as diagnostic tools [11-13]. However, in spite of growing recent interest [12], there has been no detailed statistical analysis of crackle sound. Here, we analyze lung sounds collected under conditions that promote crackle generation. We find that the distribution of crackle intensities follows a power law, which we interpret using a model of crackle generation and propagation in a tree structure mimicking the bronchial geometry.

\section{EXPERIMENTAL DATA}

We measure the sound pressure field generated by airway openings in isolated dog lung lobes. We cannulate the main bronchus of the lobes, and place the lobes in an airtight chamber with the cannula attached to a metal tube which is led through the lid of the chamber. We inflate the lobes in $120 \mathrm{~s}$ from the collapsed state to total lobe capacity by creating a steadily increasing negative pressure in the chamber using a suction pump. The inlet of the main bronchus and the outlet of the suction pump are connected to separate mechanical low-pass filters to minimize environmental and pump noise in the acoustic measurements. To minimize the effects of possible reflections from the low-pass filter back to the microphone, we use a 4-m-long uniform tube with no change in its characteristic impedance. We detect the sound at the inlet of the main bronchus with a low-noise microphone, amplified and sampled at a rate of $22050 \mathrm{~Hz}$. We record chamber pressure with respect to atmospheric pressure (transpulmonary pressure) and airflow to the lobes sampled at a rate of $80 \mathrm{~Hz}$. Pressure and airflow were measured using a Valydine MP-45 transducer $\left(50 \mathrm{~cm} \mathrm{H}_{2} \mathrm{O}\right)$ and a screen pneumotachometer (resistance of $5 \mathrm{~cm} \mathrm{H}_{2} \mathrm{O} / \mathrm{l} / \mathrm{s}$ ) attached to another Validyne MP-45 transducer $\left(2 \mathrm{~cm} \mathrm{H}_{2} \mathrm{O}\right)$, respectively. We collect a total of 12 pressure-volume curves and 12 sound pressure time series during the slow inflation of 12 separate lobes. At the beginning of inflation, the sound pressure time series display a discrete set of cracklesintermittent pressure transients, each consisting of an initial negative spike followed by a short ringing [see Fig. 1(a)]. When the pressure-volume curve reaches its lower knee, massive airway openings generate dense and overlapping wave packets. The envelope of the time series gradually decreases with inflation, indicating first the generation of coarse crackles, then later of fine crackles [11]. To characterize the statistical features of the irregular crackle time series, we developed a moving window algorithm that detects and measures the size $s$ of the negative spikes in the time series. An example of the spike time series is shown in Fig. 1(b). The overall distribution $n(s)$, including data from all 12 inflation experiments, follows a power law behavior, $n(s) \propto s^{-\alpha}$, with an exponent $\alpha=2.77 \pm 0.05$ [Fig. 1(c)]. Also, in each of the 12 different inflation experiments, the power law distribution extends over nearly two orders of magnitude with similar exponents (with an average $\alpha$ $=2.76 \pm 0.19$ ).

\section{MODEL FORMULATION}

To interpret the power law distribution of the spikes, we develop a model of crackle wave generation and propagation 

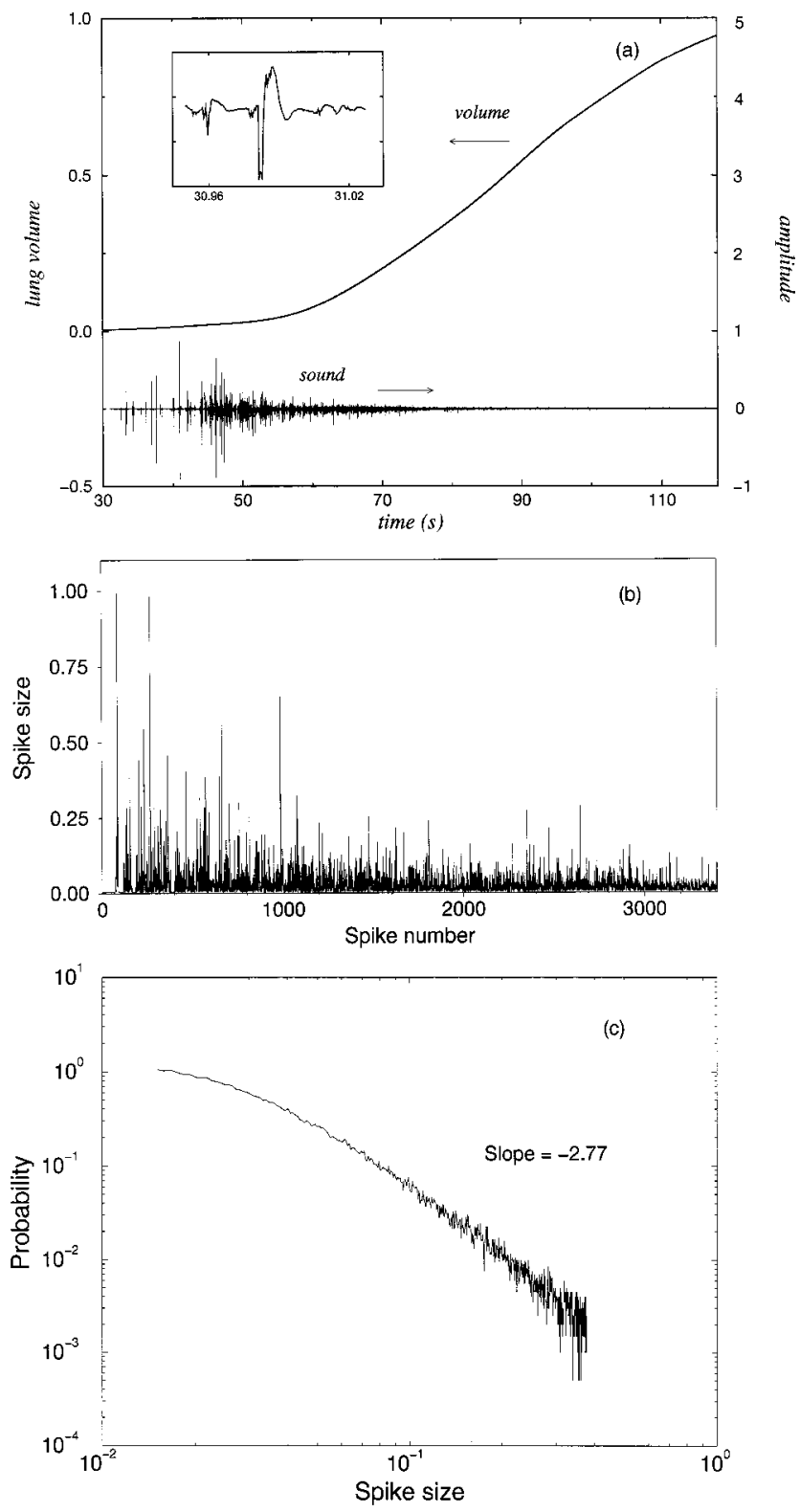

FIG. 1. (a) Time series of raw sound data as well as lung volume, both as functions of inflation time. Sound pressure is in arbitrary units, and lung volume is normalized to total lung capacity at the end of inflation. The inset is a zoom to a short segment of the sound wave, showing first a smaller, then a larger crackle waveboth consisting of an initial negative spike followed by a short ringing. (b) Time series of the normalized negative spikes for experimental data in (a). (c) Log-log plot of the size distribution $n(s)$ of spike amplitudes based on data from 12 independent inflations.

in a tree structure. When the lungs deflate to very low volumes, many peripheral airways close up by forming a liquid bridge between the collapsed airway walls [14]. Experiments on flexible tube models and in isolated lungs indicate that the opening of a single airway can be characterized by a critical opening threshold pressure $P[15]$. If the pressure at the inlet of an airway exceeds this threshold, the airway opens. Thus, we model the airway tree with a binary tree in which a threshold pressure $P_{i, j}$ is assigned to each airway $(i, j)$, where $i$ is the generation number $(i=1, \cdots, N)$ and $j \in\left[1,2^{i}\right]$. The distribution of $P_{i, j}$ is broad [6] and will be examined below. We assume that at time $t=0$, all airways are initially blocked. The inflation is simulated by applying an external pressure $P_{E}$ at the top of the tree. $P_{E}$ is initially assigned the value $P_{0,0}$, the threshold pressure of airway $(0,0)$. Since an airway opens when the pressure in its parent equals or exceeds its critical threshold pressure, the airway $(0,0)$ now opens and its pressure is set equal to $P_{E}$. Next, the two daughter airways $(1,0)$ and $(1,1)$ are checked; if $P_{E}$ $\geqslant P_{1,0}$ then $(1,0)$ opens and if $P_{E} \geqslant P_{1,1}$ then $(1,1)$ also opens. This opening process is then continued sequentially down the tree until no airway is found with $P_{i, j} \leqslant P_{E}$. This process defines a cascade or avalanche of opening [5]. The inflation is then continued by gradually increasing $P_{E}$ in small increments until the entire tree becomes open. We next describe how crackle waves are generated in individual airways and how they propagate up the tree. Before the opening of an airway occurs, a large pressure gradient builds across the fluid plug that blocks the segment. When $P_{E}$ reaches $P_{i, j}$ of the airway, the meniscus of the fluid plug is either rapidly pushed out of the segment or disrupted. This process is fast [15] and the pressure gradient is sufficiently steep to generate a transient sound wave-a crackle. We assume that this sound wave can be represented as a single spike with an amplitude $s_{i, j}$ proportional to the local threshold pressure $P_{i, j}$. This sound wave travels in both directions, but we are only interested in the wave traveling toward the root of the tree. A wave traveling up in a daughter branch will be attenuated due to several factors. Here we consider attenuation due to a change in geometry at a bifurcation. Based on continuity of pressure and volume velocity at the bifurcation, the original pressure spike amplitude $s_{i, j}$ will be attenuated as [16]

$$
s_{i, j}^{1}=\frac{2 A_{i, j}}{A_{i, j}+A_{i, j+1}+A_{i-1, j / 2}} s_{i, j}=b_{i, j} s_{i, j},
$$

where $s_{i, j}^{1}$ is the pressure spike amplitude transmitted from the branch $(i, j)$ into its parent branch $(i-1, j / 2)$. The other daughter of the parent branch is denoted by $(i, j+1)$, and $A$ denotes the cross-sectional area of a branch. The factor $b_{i, j}$ is the acoustic attenuation coefficient that depends on the local geometry. When the wave passes through a cascade of $i$ bifurcations to reach the root, the total attenuation is obtained by successively applying the above equation at each bifurcation. When an avalanche is initiated, each segment participating in the avalanche generates a crackle wave locally, which is then propagated up the tree and the waves are superimposed at the root to simulate the measured spike time series. We can obtain an analytical distribution of the sound pressure spikes, if we also assume that $b_{i, j}=b(b=\mathrm{a}$ constant throughout the tree), and all $P_{i, j}=1$. Hence all the local $s_{i, j}=1$. In this case, a wave initiated at generation $i$ will pass $i$ bifurcations to arrive at the root with an amplitude $s=b^{i}$. Thus, the distribution $n(s)$ will be taking discrete values of $b^{0}, b^{1}, \ldots, b^{N}$. Since at generation $i$ we have $2^{i}$ segments each sending a wave up the tree, the corresponding number of spikes are $2^{0}, 2^{1}, \ldots, 2^{N}$. On a log-log graph, this defines a power law. By normalizing the histogram with the bin size, $b^{i-1}-b^{i}$, the exponent of the tail of the probability distribution $n(s)$ is 


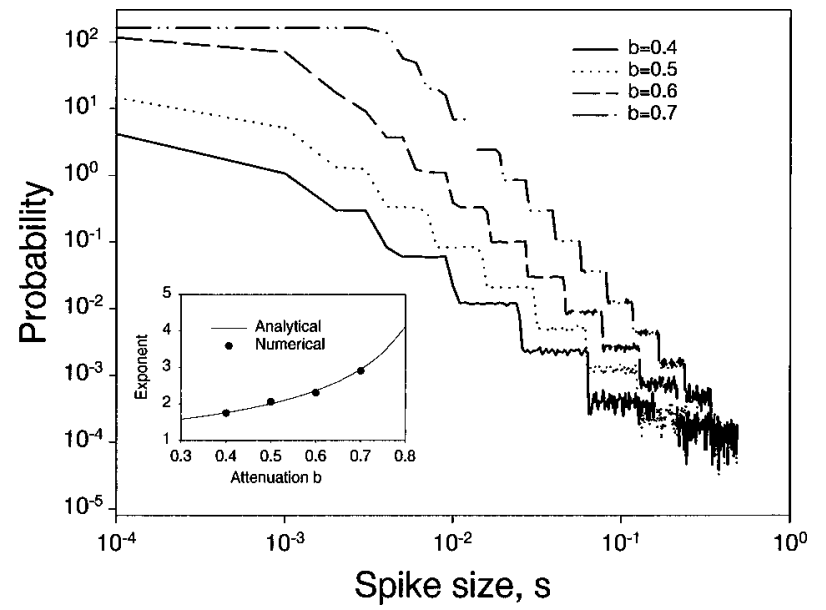

FIG. 2. The distribution $n(s)$ of spike sizes in a binary tree model of $N=15$ generations for different values of the attenuation $b$. The threshold pressure distribution is uniform. The inset compares the analytical exponent (solid line) and the exponent estimated from the envelope of the distributions.

$$
\alpha=\frac{\ln (2 / b)}{\ln (b)} .
$$

\section{NUMERICAL AND ANALYTICAL RESULTS}

Next, we examine numerically how $n(s)$ and $\alpha$ change when we relax some of the simplifying assumptions. In Fig. 2 , we show the effect of distributing $P_{i, j}$ uniformly between 0 and 1 independent of generation number. The distribution $n(s)$ is now a staircase, and the overall slope is sensitive to $b$. However, we find good agreement between Eq. (2) and the exponent of the envelope of the distributions (see inset). When the value of $b$ is not constant but has a certain probability distribution with mean $\bar{b}$ and standard deviation $\sigma$, the staircase gradually disappears, but $\alpha$ does not depend on $\sigma$, only on $\bar{b}$. Figure 3(a) shows how $n(s)$ depends on the system size for $N=10,14$, and 18 . Increasing the number of generations increases the saturation value of $n(s)$ and decreases the crossover spike size $s_{\times}$, and hence the scaling region of $n(s)$ is shifted toward lower values of $s$. However, the exponent $\alpha$ remains the same for all $N$. Assuming that $P_{i, j}$ and hence the wave amplitudes $s_{i, j}$ are uniformly distributed, we can derive the distribution function for the wave amplitude at the root as follows. Let us denote the probability by $n_{i}(s)$ that a wave initiated at generation $i$ has a size $s$. Since $s_{i, j}$ are uniformly distributed between 0 and $1, n_{i}(s)$ is also uniformly distributed, but between 0 and $b^{i}$. Due to normalization of the distribution, the value of this probability is $b^{-i}$. We are interested in the probability of finding a spike size $s$ at the root that can come from any generation $i$ $=1, \ldots, N$. This probability, $n(s)$, will be the weighted average of the probabilities $n_{i}(s)$ for $i=1, \ldots, N$. The weights are the relative number of segments at each generation, i.e., $2^{i} / Q$ where $Q=2^{0}+2^{1}+\ldots+2^{N}$. This again defines a power law on the log-log graph of $n(s)$ and $s$ with a slope $\alpha$ given by Eq. (2). Summing the geometric series, we obtain a closed form approximation,
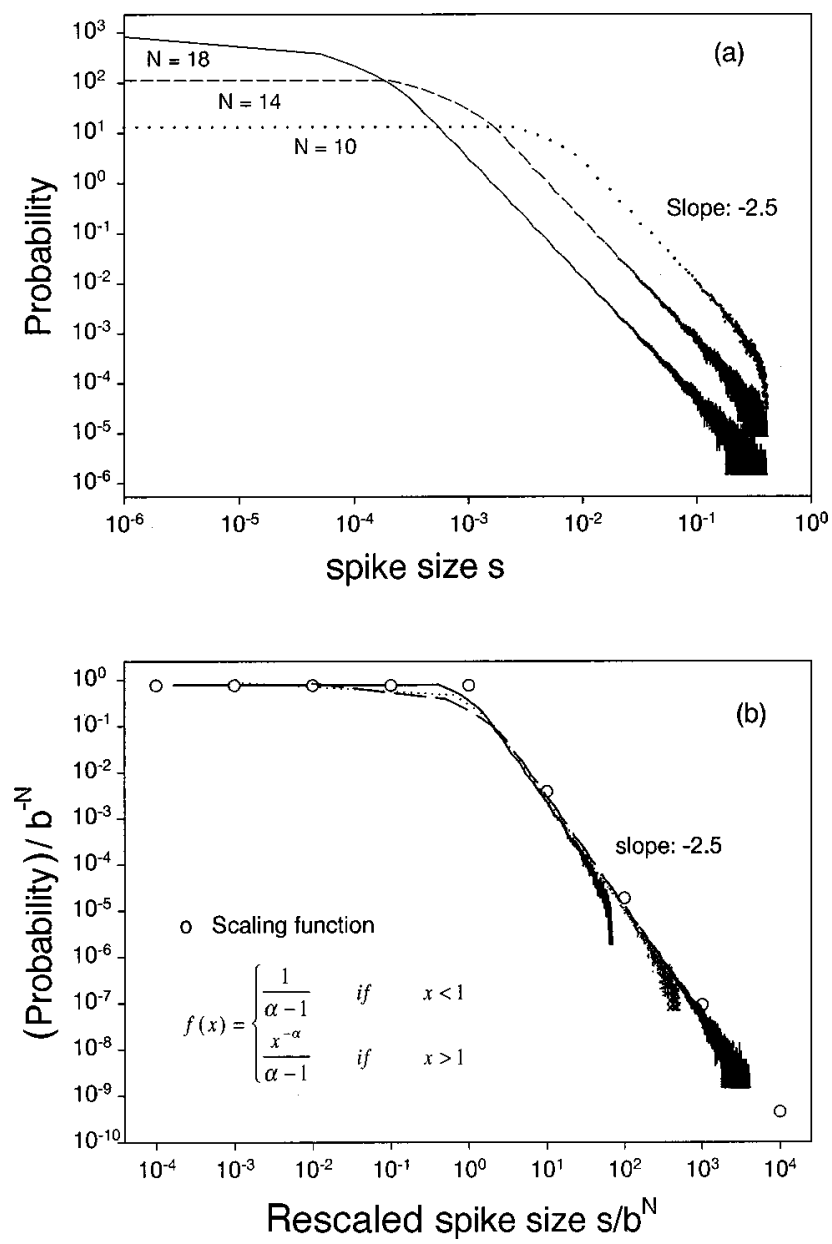

FIG. 3. (a) The distribution of spike sizes as a function tree size $N$. The mean attenuation $\bar{b}$ is 0.6 and the threshold pressure distribution is uniform. (b) Renormalization of the spike size distributions corresponding to $N=10,14$ and 18 shown in (a). The open circles denote the analytical scaling function.

$$
n(s)=\frac{1}{b^{N}} f\left(\frac{s}{b^{N}}\right)
$$

where $f(x)$ is a scaling function

$$
f(x)=\left\{\begin{array}{cl}
\frac{1}{\alpha-1} & \text { if } x<1 \\
\frac{x^{-\alpha}}{\alpha-1} & \text { otherwise. }
\end{array}\right.
$$

Equation (4) implies that the crossover from a flat to a power-law behavior of $n(s)$ occurs at $s_{\times}=b^{N}$ with a saturation value of $b^{-N}$. Thus, rescaling $s$ by $b^{N}$ and $n(s)$ by $b^{-N}$, we can collapse all the curves in Fig. 3(a) to a single master distribution. Indeed, Fig. 3(b) demonstrates that the distributions for different $N$ fall on a single curve. The scaling function is in good agreement with the numerical simulations.

\section{DISCUSSION}

Several assumptions have been made in our model of crackle generation and propagation. First, the airway segments have been assumed to be rigid tubes. Flexible airway 
walls would be important in describing the mechanism of ringing [see Fig. 1(a)] which we do not analyze in this study. Second, we also assume that the only mechanism that produces attenuation is that of a wave passing through a bifurcation. Attenuation due to energy dissipation in the viscoelastic airway walls is not likely to be important. The reason is that the spikes carry high-frequency energy for which the airways behave nearly like rigid pipes. Additionally, attenuation in air is even less than attenuation in tissue. Following the generation of a wave, we assume that a single wave travels up the tree and that wave reflection does not affect the spike size distribution. When the original wave passes the first bifurcation toward the root, it will be reflected. The reflected wave will be re-reflected from the first bifurcation downward and this second reflection will follow the original wave. The secondary waves, however, do not affect the spike size distribution. The lengths of the small airways range from 0.1 to $0.3 \mathrm{~cm}$. The sound wave speed in small airways is $268 \pm 44 \mathrm{~m} / \mathrm{s}$ [17]. An upper limit for the time difference between the original and the reflected wave is $(0.3 \mathrm{~cm}) /(200 \mathrm{~m} / \mathrm{s})=0.000015 \mathrm{~s}$. The smallest time difference between two spikes we can identify is only $2 / 22050 \approx 0.0001 \mathrm{~s}$. Thus, the first reflection cannot be separated from the original wave going upward. There should be at least six consecutive reflections to achieve a time delay that can be reliably measured with our system. At a bifurcation, the reflected energy would be less than $35 \%$ of the incident energy. After 6 reflections, the amplitude decreases to below $0.2 \%$ of the original wave amplitude. Numerical simulations show that reflections having amplitudes up to $10 \%$ of the original waves do not influence the scaling behavior. We assume that airways open via avalanches. This is important since, as we explain below, it leads to the intermittent large pressure spikes in the crackle time series. These large pressure spikes are generated when pressure gradually builds up in front of a segment due to a local high threshold pressure. When this threshold pressure is overcome by the external pressure field, the segment opens, initiating an avalanche in which many segments below the first segment sequentially open. Every segment that opens in an avalanche generates a crackle. The crackle amplitudes that are sequentially generated within an avalanche must be on average smaller and smaller as they come from deeper regions and the crackle waves are attenuated by more and more bifurcations. This process leads to the observed intermittent behavior of the spike time series. An important assumption regarding the model structure is that the tree is symmetric. Using similar arguments as above, one can show that the scaling behavior does not change if the binary tree is incomplete, i.e., asymmetric. In a symmetric tree, the number of branches at generation $i$ is $2^{i}$ whereas in an asymmetric tree the number of branches at generation $i$ is $a^{i}$, where $a<2$. Thus, the exponent in Eq. (2) changes to $\alpha=\ln (a / b) / \ln (b)$ where the asymmetry parameter takes the value of $a=2$ for a symmetric tree. Physiologically realistic asymmetry is obtained for $a=1.95$, which decreases $\alpha$ by 5\%. Another important assumption is that the threshold pressures are independent of generation. The effect of introducing a distribution for $P_{i, j}$ that slightly depends on the generation number to reflect the fact that threshold pressures are, on average, an increasing function of decreasing diameter [15] (and hence of increas-
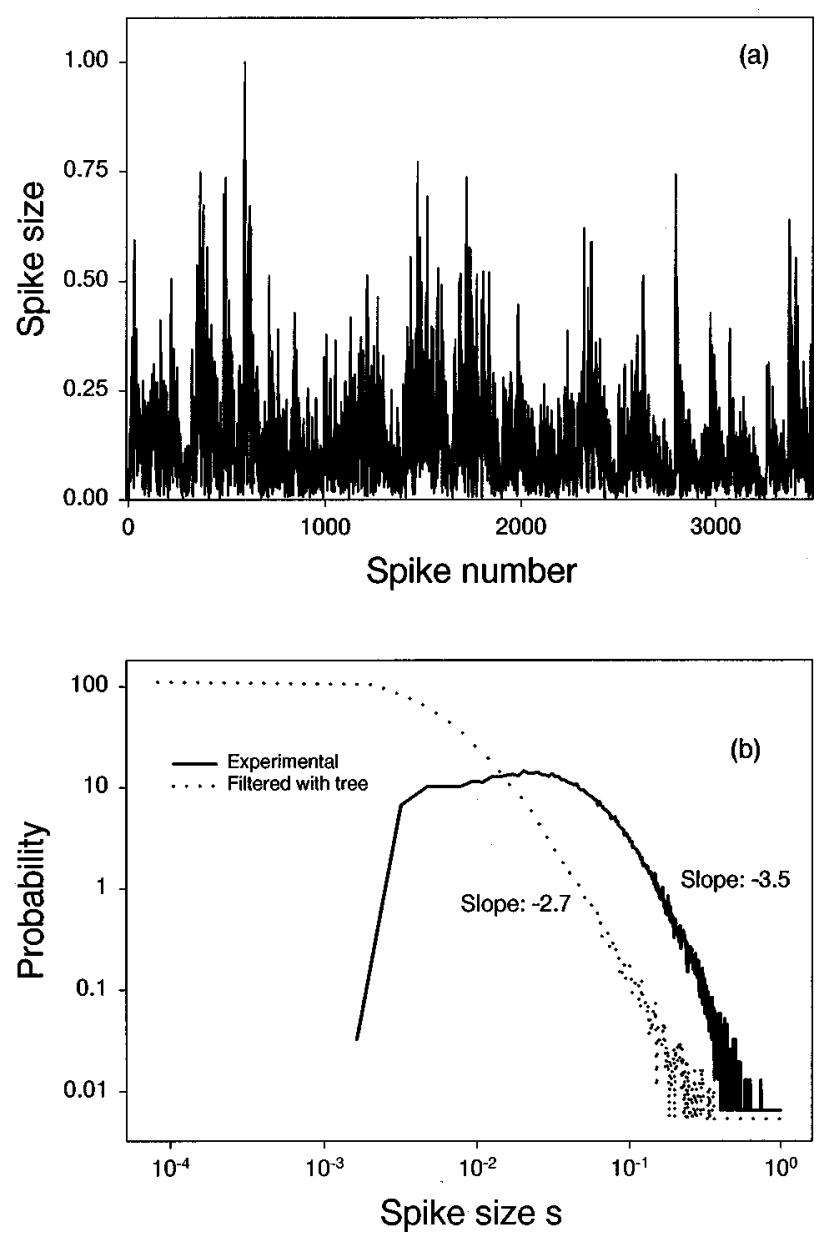

FIG. 4. (a) Time series of sound pressure spikes of bubbling champagne. (b) Solid line shows the log-normal-like distribution of the time series in (a). Dotted line is the distribution after passing the time series in (a) through the tree structure.

ing generation number), is to increase $\alpha$ by no more than $10 \%$. However, the scaling behavior is again not affected. Thus, we find that it is the tree structure that produces scaling behavior in the crackle size distribution. To further test that the origin of scaling in lung crackle sound is the tree structure of the airways, we measure the bubbling sound of a gaseous control fluid (sparkling wine). Using a similar setup, we place the microphone about $2 \mathrm{~cm}$ above the surface of the control fluid in a glass. We apply the same spike detection algorithm to the sound pressure to identify the spike time series shown in Fig. 4(a). The spike time series from the control fluid is qualitatively different from that in Fig. 1(b). The distribution of the spike sizes in Fig. 4(b) is similar to a log-normal distribution unlike the power law distribution found for the lung [see Fig. 1(c)]. When we use the local sound spike time series of the control fluid as input to our tree model, the sound pressure distribution at the root of the tree is indistinguishable from that of the real lung sound distribution [see dashed line in Fig. 4(b)]. Thus, the tree structure acts as a strong acoustic filter such that the sound pressure distribution at the root becomes a power law independent of the nature of the locally generated sound.

\section{CONCLUSION}

With regard to the physiological implications, rescaling the experimental data to fit the scaling function of Eq. (2) 
requires that $b=0.65$ and $N=15$, whereas the value of $b$ estimated from independent morphometric airway dimensions is 0.62 , which is close to our estimate obtained from dynamic sound measurements. The tree size $N=15$ is also a reasonable estimate for the number of airway generations initially closed in a collapsed lung [18]. The quantitative agreement between the spike time series from the experiments and simulations suggests that (i) the irregularities are a consequence of the heterogeneity in the threshold pressures and airway structure of the lung, (ii) the intermittent behavior of the crackle spike time series is due to the avalanchelike opening of the airway segments, and (iii) the scaling behavior is a result of the successive attenuations acting on the sound spikes as they propagate through a cascade of bifurcations along the airway tree. Finally, we note that the char- acteristics of crackles have long been used as diagnostic tools to differentiate among a variety of pulmonary diseases. However, the use of crackles in diagnosis has been based on medical experience collected over many years. Our results here provide a physical understanding of crackles and will allow us to estimate the average diameter of the airways where crackles come from. This has potential clinical importance, since it may allow the localization of closed airways, and, hence, of local edema or inflammation of tissue.

\section{ACKNOWLEDGMENTS}

We thank P. Ch. Ivanov for helpful discussions, and the NSF, CNPq, Hungarian Scientific Research Fund T016308, and FUNCAP for support.
[1] Fractals in Natural Sciences, edited by T. Vicsek, M. Shlesinger, and M. Matsushita (World Scientific, Singapore, 1994); B.J. West and W. Deering, Phys. Rep. 246, 1 (1994).

[2] J.B. Bassingthwaighte, L.S. Liebovitch, and B.J. West, Fractal Physiology (Oxford University Press, New York, 1994); B. J. West, Fractal Physiology and Chaos in Medicine (World Scientific, Singapore, 1990).

[3] M.F. Shlesinger and B.J. West, Phys. Rev. Lett. 67, 2106 (1991)

[4] B.J. West and M.F. Shlesinger, Int. J. Mod. Phys. B 3, 795 (1989).

[5] B. Suki, A.L. Barabási, Z. Hantos, F. Peták, and E.H. Stanley, Nature (London) 368, 615 (1994); A.-L. Barabási, S.V. Buldyrev, H.E. Stanley, and B. Suki, Phys. Rev. Lett. 76, 2192 (1996).

[6] M.K. Sujeer, S.V. Buldyrev, S. Zapperi, J.S. Andrade, Jr., H.E. Stanley, and B. Suki, Phys. Rev. E 56, 3385 (1997).

[7] P. Forgacs, Lancet 2, 203 (1967).

[8] J.J. Fredberg and S.K. Holford, J. Acoust. Soc. Am. 73, 1036 (1983).

[9] M. Munakata et al., J. Appl. Physiol. 61, 1120 (1986); T. Katila et al., ibid. 71, 2173 (1991).
[10] D.G. Frazer et al., Environ. Health Perspect. 66, 25 (1986).

[11] R.L.H. Murphy et al., N. Engl. J. Med. 296, 968 (1977).

[12] H. Pasterkamp, S.S. Kraman, and G.R. Wodicka, Am. J. Respir. Crit. Care Med. 156, 974 (1997).

[13] P. Piirilä, Chest 102, 176 (1992); A.R. Nath and L.H. Capel, Thorax 29, 695 (1974); M. Mori et al., ibid. 35, 843 (1980); A.R. Nath and L.H. Capel, ibid. 35, 694 (1980); N. Al-Jarad et al., ibid. 48, 347 (1993); P. Piirilä et al., Chest 99, 1076 (1991); Y. Ploysongsang et al., J. Appl. Physiol. 66, 2061 (1989).

[14] D.R. Otis, Ph.D. thesis, Massachusetts Institute of Technology, 1995; D.R. Otis et al., J. Appl. Physiol. 80, 2077 (1996).

[15] D.P. Gaver, R.W. Samsel, and J. Solway, J. Appl. Physiol. 69, 74 (1990); E.T. Naureckas et al., ibid. 76, 1372 (1994); D.Y.K. Yap et al., ibid. 76, 2095 (1994).

[16] L.E. Kinsler, A.R. Frey, A.B. Coppens, and J.V. Sanders, Fundamentals of Acoustics, 3rd ed. (John Wiley and Sons, New York, 1982).

[17] J. Rice, J. Acoust. Soc. Am. 82, 1139 (1987).

[18] B. Suki, J.S. Andrade, Jr., M.F. Coughlin, D. Stamenović, H.E. Stanley, M.K. Sujeer, and S. Zapperi, Ann. Biomed. Eng. 26, 608 (1998). 